\title{
Progression of cortical thinning in early Parkinson's disease.
}

N. Ibarretxe-Bilbao $(P h D)^{1,2,3,6}$ C. Junque $(P h D)^{1,2,3}$ B. Segura $(P h D)^{1,2,3}$ H. C. Baggio $(\mathrm{MD})^{1,2,3}$ M. J. Marti $(\mathrm{MD}, \mathrm{PhD})^{1,2,4} \mathrm{~F}$. Valldeoriola $(\mathrm{MD}, \mathrm{PhD})^{1,2,4}$ N. Bargallo (MD, $\mathrm{PhD})^{2,5}$ E. Tolosa $(\mathrm{MD}, \mathrm{PhD})^{1,2,4}$

${ }^{1}$ Centro de Investigación en Red de Enfermedades Neurodegenerativas (CIBERNED), Hospital Clínic de Barcelona, Catalonia, Spain.

${ }^{2}$ Institute of Biomedical Research August Pi i Sunyer (IDIBAPS), Catalonia, Spain.

3 Department of Psychiatry and Clinical Psychobiology, University of Barcelona, Catalonia, Spain.

${ }^{4}$ Parkinson's Disease and Movement Disorders Unit, Neurology Service, Institut Clínic de Neurociències (ICN), Hospital Clínic de Barcelona, Catalonia, Spain.

${ }^{5}$ Centre de Diagnòstic per la Imatge Hospital Clinic de Barcelona (CDIC), Hospital Clínic de Barcelona, Catalonia, Spain.

${ }^{6}$ Department of Methods and Experimental Psychology, Faculty of Psychology and Education, University of Deusto, Basque Country, Spain

Corresponding author: Carme Junque (PhD)

Department of Psychiatry and Clinical Psychobiology. University of Barcelona Casanova 143 (08036) Barcelona, Spain

Phone: (+34) 934024570 // Fax: (+34) 934035294 // E-mail: cjunque@ub.edu

Disclosure: This study was supported by Generalitat de Catalunya [2009SGR0836 to E.T., 2009SGR0941 to C. J), PSI2010-16174 to C. J and CIBERNED to N I-B. The authors report no conflicts of interest.

Word count: Abstract: 213; Main text: 2026

Running title: Cortical thinning in early PD 


\section{ABSTRACT}

Objective: To investigate the progression of cortical thinning and gray matter volume loss in early Parkinson's disease (PD).

Methods: Magnetic resonance imaging (MRI) and neuropsychological assessment were obtained at baseline and follow-up (mean $\pm \mathrm{SD}=35.50 \pm 1.88$ months) in a group of 16 early-PD patients (Hoehn and Yahr stage $\leq$ II and disease duration $\leq 5$ years) and 15 healthy controls matched for age, gender and years of education. FreeSurfer software was used for the analysis of cortical thickness, and for cortical and subcortical volumetric analyses. Voxel-based morphometry analysis was performed using SPM8.

Results: Compared with controls, PD patients showed greater regional cortical thinning in bilateral fronto-temporal regions as well as greater over-time total gray matter loss and amygdalar volume reduction. PD patients and controls presented similar over-time changes in cognitive functioning.

Conclusion: In early-PD patients, global gray matter loss, amygdalar atrophy and cortical thinning in fronto-temporal regions are specifically associated with the PD degenerative process.

Keywords: Parkinson's disease, cognition, longitudinal, cortical thickness. 


\section{INTRODUCTION}

Cognitive dysfunctions appear early in the course of Parkinson's disease (PD). Cross-sectional, community-based studies have reported impairments in executive functions $(1,2)$, attention and psychomotor speed $(3,4)$, memory (1-4), visuospatial functions (3) and verbal fluency $(1,2)$ in newly-diagnosed PD patients. Furthermore, longitudinal assessment of cognition in these early-PD patients shows a faster rate of cognitive decline than in control subjects $(5,6)$.

According to the neuropathological Braak stages (7), alpha-synuclein pathology ascends from the brainstem and olfactory bulb, in the early stages, to the midbrain and diencephalic nuclei, and later to the neocortex. However, magnetic resonance imaging (MRI) findings do not fully conform to this neuropathological staging. Cross-sectional imaging studies in early PD, using different methodological approaches, have found highly variable, and usually not extensive, patterns of structural degeneration. Specifically, voxel-based morphometry (VBM) and volumetric techniques comparing early-PD patients and matched controls have shown white matter (WM) loss in the brainstem (8) and GM loss in the striatum (9), amygdala (10) and orbitofrontal cortex (OFC) (10). Cortical thickness (CTh) studies, on the other hand, have described focal cortical thinning in the OFC, ventrolateral prefrontal cortex, occipito-parietal cortex (9) and in the supplementary motor area (11). These discrepant findings highlight the need for longitudinal studies using comprehensive techniques to help clarify the typical patterns of evolution of structural changes that take place early in Parkinson's disease.

The relationship between structural MRI changes and neuropsychological deficits in Parkinson's disease has not been extensively investigated, and the only available results come from cross-sectional studies (12). PD patients with mild cognitive impairment have been found to have fronto-temporal neocortical reductions compared with PD patients with normal cognition (13), and memory deficits have been seen to correlate with the degree of hippocampal atrophy (14-16). Similarly, correlations have been found between visuospatial and visuoperceptual deficits and 
parieto-temporal gray matter reductions (17), between verbal fluency and frontal and temporal cortical atrophy (18). Specifically for early PD, Ibarretxe-Bilbao et al. (2009) showed amygdalar and bilateral OFC degeneration associated with impaired recognition of emotions, and GM volume loss in left lateral OFC related to decisionmaking impairment (10).

VBM and cortical thickness analyses are sensitive to different though overlapping GM parameters that are altered in neurodegenerative processes: VBM provides a mixed measure of cortical GM, including cortical surface area and/or cortical folding as well as cortical thickness (19), whereas cortical thickness analysis has the advantage of providing a quantitative value that represents a physical property of the cortical mantle. These MRI techniques are considered to provide complementary information in different research settings (20-22).

To our knowledge, no published MRI studies have focused on the progression of brain changes using cortical thickness and VBM analyses in early Parkinson's disease. Indeed, longitudinal MRI studies have only been carried out at more advanced disease stages, showing progressive ventricular dilation $(23,24)$ and progressive cerebral GM loss in non-demented PD $(25,26)$, PD patients at risk for dementia (patients with visual hallucinations) (27) and in patients with PD dementia $(26,28)$.

In the present study, we have performed a prospective assessment of a group of early-PD patients and a matched control group of healthy subjects. Subjects underwent MRI and neuropsychological assessment both at study entry and after a mean follow-up interval of 35.5 months. In a previous study, we reported MRI findings at baseline showing GM volume loss in the amygdala and the OFC in early-PD patients (10) using a region-of-interest VBM approach. In the current study, we aimed to make a global assessment of the brain changes that take place over time in the early stages of PD using cortical thickness analysis, whole-brain VBM and volumetric analysis of the ventricular system and subcortical gray matter structures. We also aimed to investigate 
cognitive changes over time to establish whether these brain MRI measures correspond to possible neuropsychological changes.

\section{METHODS}

\section{Participants}

Patients were recruited from an outpatient movement disorders clinic (Parkinson's Disease and Movement Disorders Unit, Department of Neurology, Hospital Clinic, Barcelona). Healthy subjects were recruited from friends and spouses of patients and matched for age, gender and years of education. At baseline, 24 earlyPD patients and 24 healthy subjects matched for age, gender, and years of education participated in the study (the same sample used in the study by Ibarretxe-Bilbao et al., 2009 (10)). After a mean interval of 35.50 months (SD = 1.85; range 31-40 months), 16 PD patients and 15 healthy controls from this initial sample participated in the follow-up assessment. There were no significant differences between groups in terms of age, gender and education. Demographical data of the participants at baseline examination is summarized in table 1.

At the time of initial evaluation (baseline evaluation), the inclusion criteria for participating in the study were: i) fulfillment of the UK PD Society Brain Bank (PDSBB) diagnostic criteria for PD (29); ii) Hoehn and Yahr stage $\leq \mathrm{II}$; iii) disease duration $\leq 5$ years. Motor symptoms were assessed by means of the UPDRS-III motor section. Exclusion criteria were: i) presence of dementia diagnosed by a neurologist according to the Movement Disorder Society diagnostic criteria for Parkinson's disease dementia (30); ii) presence of other neurological or psychiatric disorders such as depression, defined by a Beck's Depression Inventory (BDI-II) score higher than 14 (31); and iii) the presence of visual hallucinations assessed by the Neuropsychiatric Inventory Questionnaire (NPI-Q) (32). 
This study was approved by the institutional ethics committee. Written informed consent was obtained from all study subjects after full explanation of the procedures involved.

\section{Neuropsychological assessment}

The neuropsychological assessment included the cognitive functions usually impaired in PD. The tests used were: forward and backward digit span from the Wechsler Adults Intelligence Scale (WAIS-III) as measures of immediate and working memory respectively; ii) verbal memory with Rey's Auditory Verbal Learning Test (RAVLT) as a measure of declarative memory; iii) phonemic and semantic fluencies; iv) psychomotor speed and inhibition control with the Stroop test; and v) visuomotor tracking and cognitive flexibility with the Trail Making test (TMT) (parts A and B). The neuropsychological battery was administered by a trained neuropsychologist (N.I-B), and completed in a single session lasting between one and two hours. The neuropsychologist in charge of the assessment was the same at baseline and follow-up examinations.

\section{Neuropsychological and clinical statistical analysis}

Statistical analyses of neuropsychological and clinical variables were carried out using the statistical package PASW-18 (SPSS, Inc., 2009, Chicago, IL, www.spss.com). Changes in clinical and neuropsychological variables between baseline and follow-up were analyzed using the general linear mixed model (GLM) for repeated measures to test whether these variables differed across time between groups. A two-factor repeated-measures analysis of variance $(2 \times 2)$ (RMANOVA) was performed for each of the variables. Factors were: group (PD patients and controls) and time (baseline and follow-up). Since groups were matched - there were no significant intergroup differences for age, gender and education -, these variable were 
not included in the model. The interval between neuropsychological assessments in months, on the other hand, was entered as a covariate in the RMANOVA.

\section{MRI acquisition}

Structural images at baseline and follow-up were acquired using a MAGNETOM Tim Trio 3T scanner (Siemens, Germany). High-resolution 3-dimensional T1-weighted images were acquired in the sagittal plane with an MPRAGE sequence (TR/TE 2300/2.98 ms; TI 900ms; 256×256 matrix, 1mm isotropic voxel).

\section{Cortical thickness analysis}

Cross-sectional evaluation (to determine whether PD patients and controls regionally differ in cortical thickness at baseline) and longitudinal analysis (to compare CTh between the two timepoints (baseline and follow-up)) were performed using FreeSurfer (version 5.1, available at: http://surfer.nmr.harvard.edu). In this procedure, a cortical surface three-dimensional model of CTh is created using intensity and continuity information previously described in detail (33). The initial processing of T1 high-resolution images includes several steps, which are performed independently for each subject and each timepoint: removal of non-brain tissue (34), automated Talairach transformation, intensity normalization (35), tessellation of the gray matter/white matter boundary, automated topology correction $(36,37)$, and surface deformation to optimally place the gray matter/white matter and gray matter/cerebrospinal fluid boundaries (33). The resulting representation of cortical thickness is calculated as the distance between tissue boundaries (gray matter/white matter and gray matter/cerebrospinal fluid) (33). All surface models in our study were visually inspected for accuracy.

Subsequently, for the longitudinal processing, an unbiased within-subject template space and average image (38) is created using robust, inverse consistent registration (39). Several processing steps, such as skull stripping, Talairach 
transformations, atlas registration as well as spherical surface maps and parcellations are then initialized with common information from the withinsubject template, significantly increasing reliability and statistical power (40). Finally, both time points and the template are used to create the complete subjects directories named long run. Regional differences between groups (patients and controls) in cortical measures were assessed using vertex-by-vertex general linear model (one factor with two levels without covariates, corresponding to a Student's $t$-test statistical model). Maps were smoothed using a circularly symmetric Gaussian kernel across the surface with a FWHM of $15 \mathrm{~mm}$. Z Monte Carlo simulations with 10,000 iterations were applied to CTh maps to provide clusterwise correction for multiple comparisons (41), and results were thresholded at a corrected $p$ value of $0.05(Z=$ 1.3). In order to perform main longitudinal analyses, we automatically obtained a measure of change from longitudinal data called rate of thinning ( $\mathrm{mm} / \mathrm{year})$.

Mean rates of thinning for significant clusters were then extracted for each subject in order to perform correlational analyses with cognitive measures.

We used the same methodology to perform complementary analyses with other longitudinal measures: the temporal average (the average thickness between baseline and follow-up), the percent change (the rate of thinning divided by the baseline thickness), and the symmetrized percent change (the rate of thinning divided by the average thickness).

\section{Volumetric analysis of subcortical structures}

The automated procedure for volumetric measures of brain structures implemented in FreeSurfer (version 5.1, available at: http://surfer.nmr.harvard.edu) (42) was used to obtain the volumes of subcortical structures. Volumetric measures were automatically obtained from $7 \mathrm{GM}$ structures (thalamus, caudate, putamen, pallidum, nucleus accumbens, hippocampus, amygdala), as well as from the lateral, third and fourth ventricles and the brainstem. For simplicity, we refer to these collectively as 
"subcortical structures" although, strictly speaking, hippocampus and amygdala are not subcortical. Total cortical volume, total subcortical volume and global gray matter volume were also obtained.

All the volumes obtained were compared using PASW-18. Repeated measures analysis of variance was used to longitudinally examine changes in deep gray matter structures, brainstem, ventricles and total GM volumes. Scan number was specified as a within-subject factor "time" with two levels (baseline and follow-up MRI acquisitions), and a between-subject factor "group" with two levels (PD patients and healthy controls). All the volumetric analyses controlled for intracranial volume (ICV). No other covariables were included in the model. Partial correlation analyses were performed between change in brain volumes and cognition, also controlling for ICV.

\section{Voxel based morphometry analysis}

Image preprocessing for voxel-based morphometry analyses was done using SPM8 (http://www.fil.ion.ucl.ac.uk/spm/software/spm8/). After realignment, baseline images were rigidly co-registered to the respective subject's follow-up image. All images were then segmented into GM, WM and cerebrospinal fluid probability maps with the New Segment tool of SPM8, an extended version of the "Unified Segmentation" model (43).

In an attempt to obtain an accurate subject registration and consequently an adequate detection of within-subject longitudinal changes, subject-specific templates were created using the Diffeomorphic Anatomical Registration Through Exponentiated Lie algebra (DARTEL) (44) tool to non-linearly register each subject's GM and WM baseline and follow-up images. Baseline and follow-up scans were subsequently normalized to their respective template, using the Jacobian determinants for modulation. A group template was then created using the baseline GM and WM images (normalized to the individual template) of the entire sample, also through DARTEL. Baseline and follow-up scans then underwent a second normalization, this 
time to the group template, again applying the Jacobian determinants for modulation. A third normalization was performed to bring each subject's baseline and follow-up GM images to the MNI space using an affine transformation, and then smoothed with a Gaussian kernel of $12 \mathrm{~mm}$ full-width at half-maximum. Final voxel dimension was 1.5 $\mathrm{mm}$ isotropic. A similar preprocessing approach was recently proposed by Asami et al. (45).

The final GM maps generated as described above were analyzed in a wholebrain voxelwise fashion. For cross-sectional baseline intergroup comparisons, images were entered into a two-sample $t$ test model. For longitudinal analysis, subjects' images were entered into a full-factorial model with two factors (the two levels in the group factor were entered as independent, and the two levels in the time factor as nonindependent variables, with assumed unequal variance) to evaluate possible group $\mathrm{x}$ time interactions in GM volume.

Total intracranial volume (obtained through the sum of the native GM, WM and cerebrospinal fluid maps) was entered as a covariate of no interest in all these analyses. Subjects' interscan interval (in months) was also entered as a covariate of no interest in the interaction analyses.

Results were corrected for multiple comparisons using familywise-error correction (46), with a significance threshold set at $p<0.05$.

\section{RESULTS}

\section{Neuropsychological tests and clinical variables}

In PD patients, LEDD, Hoehn and Yahr, and UPDRS-III motor section scores did not differ significantly between baseline and follow-up assessments (see table 2).

The analysis of variance for repeated measures revealed a significant time effect on MMSE and depression scores but no significant interactions between groups and time. That is, both patients and controls showed lower scores over time, but the 
decline did not differ between groups. NPI-Q scores remained stable over time in both groups.

At baseline, PD patients showed worse cognitive performance than controls. There were significant differences in neuropsychological variables measuring working memory (backward digits, $\mathrm{t}=3.015, p=0.005)$, psychomotor speed (Stroop colors, $\mathrm{t}=$ 2.755, $p=0.010$ and words-colors, $\mathrm{t}=2.417, p=0.023$ ) and cognitive flexibility (TMTB, $t=-2.146, p=0.046)$. The longitudinal analyses also showed a significant group effect in working memory (digit span backward), psychomotor speed (Stroop colors) and cognitive flexibility (TMTB). A significant time effect was seen for psychomotor speed assessed by the Stroop test (Stroop words). The only other cognitive variable for which a time effect was observed was phonemic fluency, as a result of both groups improving their performance at follow-up, suggesting a practice effect. There was no significant interaction between time and group for any of the variables analyzed (see table 3).

\section{Cortical thickness}

At baseline, there were no significant differences in cortical thickness between PD patients and the healthy control group (data not shown). However, rate-of-thinning (mm/year) analyses showed that PD patients had significantly greater progressive cortical thinning in the following areas than healthy controls at the corrected level $(p<0.05)$ (see figure 1 and table 5):

- Right superior frontal gyrus, extending to caudal middle frontal gyrus, precentral sulcus and precentral gyrus;

- Right frontal pars opercularis and precentral gyrus;

- $\quad$ Right superior temporal gyrus extending to the adjacent temporal sulcus;

- Left caudal middle frontal gyrus extending to rostral middle frontal gyrus and precentral region;

- Left middle temporal gyrus extending to parietal cortex. 
At a more conservative correction level $(p<0.01)$, clusters in the right hemisphere remained significant: in the caudal middle frontal gyrus $\left(621.2 \mathrm{~mm}^{2} ; \mathrm{x}, \mathrm{y}, \mathrm{z}\right.$ Talairach coordinates of the maximum: $29.5,7.5,47.3$ ) and in the superior temporal gyrus (two clusters, one with $549.2 \mathrm{~mm}^{2} ; \mathrm{x}, \mathrm{y}, \mathrm{z}$ Talairach coordinates of the maximum: 43.9, -4.2 , -16.2; and the other with $494.4 \mathrm{~mm}^{2} ; \mathrm{x}, \mathrm{y}, \mathrm{z}$ Talairach coordinates of the maximum: 63.1, -31.1, 5.6). The complementary longitudinal measures analyzed showed no significant group differences in the measure temporal average. However, the measures percent change and symmetrized percent change showed patterns of thinning in frontal and temporal regions similar to those obtained by means of the rate of thinning (see Supplementary Figure 1 for the results of the symmetrized percent change).

There were no significant correlations between cognitive measures and mean rate of thinning (or between cognitive measures and the complementary longitudinal measures analyzed) in the clusters described above.

No areas of significantly higher rates of thinning in controls than in PD patients were observed.

\section{Volumetric analyses}

Table 4 shows total gray matter, total cortical and subcortical gray matter volumes (total and by structure) as well as ventricular volumes at baseline and followup. At baseline, there were no significant group differences in volumetric measures (data not shown). The loss from baseline to follow-up in subcortical deep gray matter structures besides the amygdalae was similar in both groups. We also observed a similar volume increase in the ventricular system in both groups.

Total GM volume reduction over time was greater in PD patients than in healthy controls; the time-group interaction was significant. Finally, PD patients also showed specific and significant loss in amygdalar and total cortical volumes (see table 4). Total GM volume changes correlated with Stroop words performance decline in PD patients 
$(r=0.541, p=0.037)$. Neither amygdalar volume changes nor total cortical volume reduction correlated with cognitive changes.

\section{Voxel-based morphometry}

No significant group differences in GM volume were found between healthy controls and PD patients at baseline in whole-brain analyses. Interaction analysis did not reveal any areas of differential GM loss across groups at the corrected level.

For exploratory purposes, we report the results without correction for multiple comparisons - at the uncorrected level, two clusters of greater GM volume loss in PD patients than in controls were observed: in the left inferior lateral occipital cortex (3.08 $\mathrm{cm}^{3} ; \mathrm{x}, \mathrm{y}, \mathrm{z}$ MNI coordinates of the maximum: $-22,-90,-2 ; p<0.001$, uncorrected) and in the left angular gyrus $\left(0.37 \mathrm{~cm}^{3} ; \mathrm{x}, \mathrm{y}, \mathrm{z} \mathrm{MNI}\right.$ coordinates of the maximum: $-34,-56$, 26; $p<0.001$, uncorrected). We did not find any areas of significantly greater GM volume loss in healthy controls than in PD patients.

\section{DISCUSSION}

In this longitudinal study, we have found that, compared with controls, our sample of early-PD patients presented a faster rate of cortical thinning with a bilateral fronto-temporal pattern, total gray matter and total cortical volume loss, and amygdalar atrophy after an average interval of 35 months.

The first longitudinal studies in PD only evaluated global brain measures (25) or a limited number of GM areas (28). Since then, longitudinal progression of PD has been evaluated in samples formed by older patients, and with patients who had longer disease duration. In a previous work done by our group, after a follow-up of 25 months, GM loss was observed in limbic and paralimbic structures (26). In another study, including patients with visual hallucinations, we also found limbic and paralimbic plus neocortical GM loss (27). In this work we also observed that the longitudinal GM loss correlated with several measures of cognitive decline. 
To our knowledge, this is the first study to evaluate structural progressive changes in a cohort of early-Parkinson's disease patients. Our findings indicate that the degeneration of certain areas observed in other studies may not yet be detectable in non-demented, early-PD patients. The fact that our cohort was formed by younger patients could also be contributing to the absence of detectable atrophy in areas affected both by the disease and by the aging processes, such as the hippocampus (47), as these two factors are considered to interact biologically in the process of neuronal cell loss in non-dopaminergic structures (48).

In the present study, PD patients presented lower scores in some cognitive functions compared to controls at baseline, but both groups progressed at the same rate of cognitive decline over time. Previous studies reported that longitudinal changes in neuropsychological test results in early PD are much more significant in older patients (5). This observed interaction both between age and cognitive decline in PD could be the functional manifestation of the interaction between age-related and the disease-related GM atrophy observed, though this remains speculative.

In Lewy-body diseases, cognitive changes are expected to precede detectable structural changes (49) and we have found greater structural, but no greater cognitive, over-time deterioration in PD patients compared with controls. A growing body of evidence, however, suggests that cognitive deficits in PD have two general pathophysiological bases: fronto-striatal deficits, common in the early stages of disease, related to dopaminergic activity and not associated with a higher risk of evolution to dementia; and deficits with a more posterior cortical basis, which herald further cognitive decline $(5,50)$, and which are possibly related to primary cortical Lewybody or Alzheimer-type pathology (51). Indeed, at baseline, our sample of PD patients presented deficits in neuropsychological functions related to dopamine-modulated circuits, such as working memory $(52,53)$, cognitive flexibility $(53,54)$ and psychomotor speed (55). It is not surprising that deficits with a more functional basis might not be accompanied by detectable structural deterioration. The structural changes detected 
longitudinally, on the other hand, could be related to the second mechanism of cognitive decline described above, and compensatory processes in this sample of young, early-PD patients might be preventing detectable functional repercussions.

Cross-sectional structural MRI studies with early-PD patients, as pointed in the introduction of the current article, show highly variable results, sometimes with limited or no significant gray matter changes in comparison with controls (8). One study, nonetheless, did find cortical changes in early-stage patients through cortical thickness analysis (9). In it, thickness reductions were observed in frontal areas similar to the ones we have found to degenerate more rapidly in PD patients than in controls.

Overall these results suggest that the different parameters evaluated provide complementary and related information on changes occurring in PD.

A common drawback in longitudinal studies is the loss of some of the initial subjects at follow-up. In this study, the final sample of 16 PD patients, though appropriate for longitudinal analyses, does not allow dividing them into subgroups to study relevant variables such as the presence of dyskinesias, previously related to changes in the right inferior frontal gyrus (56) or behavioral abnormalities. Also, we cannot exclude that subtle cognitive decline associated with the longitudinal structural changes described could be detected with a larger sample.

One caveat in both VBM and CTh analysis is that, due to the characteristics of the underlying neuroanatomy, gray matter and cortical thickness maps are nonstationary (57). This in turn may lead to an overestimation of cluster sizes in areas of the images that display higher smoothing, and an underestimation in rougher areas (58), affecting both the false-positive and false-negative rates of analyses that include cluster-size corrections (59). Though cluster-size inference methods to correct for these effects are sometimes used in VBM analyses $(60,61)$, the permutation clusterlevel correction implemented in cortical thickness analyses assumes images to be stationary and may thus be susceptible to such artifacts. 
In conclusion, as in other neurodegenerative diseases such as AD, our findings suggest that the pathophysiological process of PD exerts specific deleterious effects in the brain, even prior to clinical manifestations of progressive decline in cognitive function.

Figure 1. Cortical areas showing significantly higher rate of thinning ( $\mathrm{mm} / \mathrm{year}$ ) in PD patients compared with controls.

The color bar indicates the significance levels in the clusters of significantly higher thinning rate in PD patients than in healthy controls, in Z values. Results were obtained using Z Monte Carlo simulation with 10,000 iterations applied to CTh maps to provide clusterwise correction for multiple comparisons. Results were thresholded at a corrected $p$ value of $0.05(Z=1.3)$.

Supplementary Figure 1. Cortical areas showing significantly higher symmetrized percent change (SPC, in percentage per year) in PD patients compared with controls.

The color bar indicates the significance levels in the clusters of significantly higher SPC in PD patients than in healthy controls, in Z values. Results were obtained using $Z$ Monte Carlo simulation with 10,000 iterations applied to CTh maps to provide clusterwise correction for multiple comparisons. Results were thresholded at a corrected $p$ value of $0.05(Z=1.3)$.

\section{AUTHOR ROLES:}


1. Research project: A. Conception, B. Organization, C. Execution;

2. Statistical Analysis: A. Design, B. Execution, C. Review and Critique;

3. Manuscript: A. Writing of the first draft, B. Review and Critique

Ibarretxe-Bilbao: $1 \mathrm{~A}, 1 \mathrm{~B}, 1 \mathrm{C}, 2 \mathrm{~A}, 2 \mathrm{~B}, 3 \mathrm{~A}$; Junque: $1 \mathrm{~A}, 1 \mathrm{~B}, 1 \mathrm{C}, 2 \mathrm{~B}, 2 \mathrm{~A}, 2 \mathrm{C}, 3 \mathrm{~B}$; Marti:

1B, 1C, 2C, 3B; Valldeoriola: 1C, 2C, 3B; Segura: 1C, 2B, 2C, 3B; Baggio: 1C, 2B,

2C, 3B; Bargallo: 1C, 2C, 3B; Tolosa: 1B, 1C, 2C, 3B

FINANCIAL DISCLOSURE: Ibarretxe-Bilbao: Grants: Spanish Ministry of Education and Science (AP 2005-019), Center of Biomedical Investigation in Neurodegenerative Diseases (CIBERNED), Employment: Full Professor University of Deusto; Junque: Grants: Spanish Ministry of Education and Science (Spanish Ministry of Education and Science PSI2010-16174, Catalonia Government 22009SGR94, Intellectual Property Rights: Books Editorial: Masson, Sintesis, Glosa, Ariel, Ars Medica, Employment: Full Professor University of Barcelona; Marti: Grants: Fundació la Marató de TV3 2006" (N2006-TV060510), Employment: Hospital Clínic i Provincial de Barcelona; Valldeoriola: Grants: FIS (Instituto de Salud Carlos III), Advisory boards: Boehringer, Ingelheim, Solvay. Employment: Hospital Clínic i Provincial de Barcelona; Segura: Grants: Spanish Ministry of Education and Science PSI2010-16174; Catalonia Government, 22009SGR94; Baggio: Grants: Spanish Ministry of Education and Science PSI201016174; Catalonia Government, 22009SGR94; Bargallo: Employment: Hospital Clínic i Provincial de Barcelona; Tolosa: Grants: Center of Biomedic Investigation in Neurodegenerative Diseases (CIBERNED), Employment: Hospital Clínic i Provincial de Barcelona, University of Barcelona. 
Table 1. Demographical data at baseline.

\begin{tabular}{|l|l|l|l|l|}
\hline & $\mathrm{PD}(\mathrm{n}=16)$ & Controls $(\mathrm{n}=15)$ & $\mathrm{t}^{\mathrm{a}}, \mathrm{X}^{\mathrm{b}}$ & $\mathrm{p}$ \\
\hline Age & $\begin{array}{l}55.94 \\
(8.10)\end{array}$ & $\begin{array}{l}57.73 \\
(9.53)\end{array}$ & $0.567^{\mathrm{a}}$ & 0.575 \\
\hline Gender (male/female) & $12 / 4$ & $12 / 3$ & $0.111^{\mathrm{b}}$ & 0.539 \\
\hline Education (years) & $\begin{array}{l}11.00 \\
(4.86)\end{array}$ & $\begin{array}{l}12.60 \\
(3.33)\end{array}$ & $1.075^{\mathrm{a}}$ & 0.298 \\
\hline Age onset & $\begin{array}{l}52.97 \\
(8.24)\end{array}$ & - & - & - \\
\hline Disease duration (years) & $\begin{array}{l}2.97 \\
(1.45)\end{array}$ & - & - & - \\
\hline
\end{tabular}

Values are mean (SD). PD= Parkinson's disease.

a Student's t-test, ${ }^{\mathrm{b}}$ chi-squared test statistics. 
Table 2. Clinical data at baseline and at follow-up and time effect, group effect, and interactions between time and group.

\begin{tabular}{|l|l|l|l|l|l|l|l|}
\cline { 2 - 8 } \multicolumn{1}{c|}{} & \multicolumn{2}{l|}{ PD (n=16) } & \multicolumn{2}{l|}{ Controls (n=15) } & \multirow{2}{*}{ F(T) } & F(G) & F(GXT) \\
\cline { 2 - 8 } \multicolumn{1}{c|}{} & Baseline & Follow-up & Baseline & Follow-up & & \\
\hline MMSE & $\begin{array}{l}29.56 \\
(0.51)\end{array}$ & $\begin{array}{l}27.94 \\
(2.62)\end{array}$ & $\begin{array}{l}29.87 \\
(0.35)\end{array}$ & $\begin{array}{l}28.80 \\
(0.68)\end{array}$ & $17.358^{\star}$ & 1.185 & 0.172 \\
\hline BDI-II & $\begin{array}{l}7.69 \\
(5.44)\end{array}$ & $\begin{array}{l}5.81 \\
(4.92)\end{array}$ & $\begin{array}{l}4.93 \\
(5.80)\end{array}$ & $\begin{array}{l}2.53 \\
(2.47)\end{array}$ & $4.440^{\star}$ & 3.281 & 0.035 \\
\hline NPI & $\begin{array}{l}3.31 \\
(5.50)\end{array}$ & $\begin{array}{l}2.00 \\
(1.97)\end{array}$ & $\begin{array}{l}1.53 \\
(1.88)\end{array}$ & $\begin{array}{l}2.33 \\
(3.58)\end{array}$ & 0.168 & 1.099 & 0.960 \\
\hline UPDRS-III & $\begin{array}{l}15.44 \\
(3.71)\end{array}$ & $\begin{array}{l}15.38 \\
(4.35)\end{array}$ & & & 0.069 & & \\
\hline $\begin{array}{l}\text { Hoehn } \\
\text { \& Yahr }\end{array}$ & $\begin{array}{l}1.81 \\
(0.31)\end{array}$ & $\begin{array}{l}1.91 \\
(0.38)\end{array}$ & & & 0.285 & & \\
\hline LEDD & $\begin{array}{l}282.50 \\
(317.98)\end{array}$ & $\begin{array}{l}444.38 \\
(357.50)\end{array}$ & & & 0.721 & & \\
\hline
\end{tabular}

*Significant at $p$ value $<0.05$

The $F$ values refer to those obtained through a repeated-measures general linear model. $F(T)$ : time effect; $F(G)$ : group effect; $F(G \times T)$ : Interaction between time and group.

MMSE: Mini-mental state examination; BDI-II: Beck Depression Inventory II; NPI: Cumming's Neuropsychiatric Inventory; UPDRS: Unified Parkinson's disease rating scale; LEDD: Levodopa equivalent daily dose, in mg. 
Table 3. Neuropsychological data at baseline and at follow-up. Time effect, group effect and interaction between time and group

\begin{tabular}{|c|c|c|c|c|c|c|c|}
\hline & \multicolumn{2}{|c|}{$P D(n=16)$} & \multicolumn{2}{|c|}{ Controls $(n=15)$} & \multirow{2}{*}{$F(T)$} & \multirow{2}{*}{$F(G)$} & \multirow{2}{*}{$\mathrm{F}(\mathrm{GxT})$} \\
\hline & Baseline & Follow-up & Baseline & Follow-up & & & \\
\hline \multicolumn{8}{|c|}{ Attention, immediate recall and working memory } \\
\hline $\begin{array}{l}\text { Digit span } \\
\text { (forward) }\end{array}$ & $\begin{array}{c}7.94 \\
(1.61)\end{array}$ & $\begin{array}{c}8.25 \\
(2.05)\end{array}$ & $\begin{array}{c}9.00 \\
(1.31)\end{array}$ & $\begin{array}{c}8.33 \\
(2.29)\end{array}$ & 0.310 & 0.617 & 3.285 \\
\hline $\begin{array}{l}\text { Digit span } \\
\text { (backward) }\end{array}$ & $\begin{array}{l}5.38 \\
(1.78)\end{array}$ & $\begin{array}{l}5.69 \\
(1.92)\end{array}$ & $\begin{array}{l}7.07 \\
(1.28)\end{array}$ & $\begin{array}{l}7.27 \\
(2.28)\end{array}$ & 0.890 & $5.275^{\star}$ & 0.029 \\
\hline Digits total & $\begin{array}{l}13.31 \\
(3.01)\end{array}$ & $\begin{array}{l}13.93 \\
(3.47)\end{array}$ & $\begin{array}{l}16.07 \\
(2.40)\end{array}$ & $\begin{array}{l}15.60 \\
(4.35)\end{array}$ & 0.021 & 2.769 & 1.239 \\
\hline $\begin{array}{l}\text { WM (direct } \\
\text { minus inverse) }\end{array}$ & $\begin{array}{c}2.56 \\
(1.56)\end{array}$ & $\begin{array}{c}2.69 \\
(2.15)\end{array}$ & $\begin{array}{l}1.93 \\
(0.96)\end{array}$ & $\begin{array}{c}1.00 \\
(1.31)\end{array}$ & 1.327 & $6.280^{\star}$ & $4.452^{*}$ \\
\hline \multicolumn{8}{|l|}{ Verbal memory } \\
\hline $\begin{array}{l}\text { RAVLT } \\
\text { learning }\end{array}$ & $\begin{array}{c}45.38 \\
(11.73)\end{array}$ & $\begin{array}{c}44.06 \\
(10.29)\end{array}$ & $\begin{array}{l}50.60 \\
(8.41)\end{array}$ & $\begin{array}{l}48.53 \\
(9.44)\end{array}$ & 0.268 & 2.072 & 0.248 \\
\hline $\begin{array}{l}\text { RAVLT } \\
\text { Delayed Recall }\end{array}$ & $\begin{array}{c}8.88 \\
(3.40)\end{array}$ & $\begin{array}{c}9.67 \\
(3.20)\end{array}$ & $\begin{array}{l}10.33 \\
(2.26)\end{array}$ & $\begin{array}{c}9.87 \\
(2.72)\end{array}$ & 0.143 & 1.444 & 2.462 \\
\hline $\begin{array}{l}\text { RAVLT } \\
\text { Recognition }\end{array}$ & $\begin{array}{l}27.69 \\
(2.27)\end{array}$ & $\begin{array}{l}28.56 \\
(2.13)\end{array}$ & $\begin{array}{l}28.40 \\
(1.88)\end{array}$ & $\begin{array}{l}28.40 \\
(1.80)\end{array}$ & 1.851 & 0.008 & 3.342 \\
\hline $\begin{array}{l}\text { RAVLT } \\
\text { True } \\
\text { recognition }\end{array}$ & $\begin{array}{l}13.68 \\
(1.70)\end{array}$ & $\begin{array}{l}14.00 \\
(1.67)\end{array}$ & $\begin{array}{l}14.20 \\
(0.86)\end{array}$ & $\begin{array}{l}14.07 \\
(1.22)\end{array}$ & 0.179 & 0.019 & 1.257 \\
\hline $\begin{array}{l}\text { RAVLT } \\
\text { False } \\
\text { recognition }\end{array}$ & $\begin{array}{c}1.00 \\
(1.21)\end{array}$ & $\begin{array}{c}0.44 \\
(0.73)\end{array}$ & $\begin{array}{c}0.73 \\
(1.62)\end{array}$ & $\begin{array}{c}0.67 \\
(0.90)\end{array}$ & 1.310 & 0.234 & 1.974 \\
\hline \multicolumn{8}{|l|}{ Verbal fluencies } \\
\hline $\begin{array}{l}\text { Phonemic } \\
\text { fluency }\end{array}$ & $\begin{array}{l}13.88 \\
(5.19)\end{array}$ & $\begin{array}{l}15.93 \\
(6.71)\end{array}$ & $\begin{array}{l}15.73 \\
(4.95)\end{array}$ & $\begin{array}{l}16.93 \\
(4.79)\end{array}$ & $52.659^{*}$ & 0.602 & 0.001 \\
\hline $\begin{array}{l}\text { Semantic } \\
\text { fluency }\end{array}$ & $\begin{array}{l}18.19 \\
(4.71)\end{array}$ & $\begin{array}{l}17.19 \\
(5.71)\end{array}$ & $\begin{array}{l}20.47 \\
(5.25)\end{array}$ & $\begin{array}{l}19.80 \\
(4.90)\end{array}$ & 1.031 & 1.661 & 2.192 \\
\hline \multicolumn{8}{|c|}{ Psychomotor speed and inhibition control } \\
\hline $\begin{array}{l}\text { SCWT Word } \\
\text { (W) }\end{array}$ & $\begin{array}{l}100.19 \\
(18.74)\end{array}$ & $\begin{array}{c}92.19 \\
(19.30)\end{array}$ & $\begin{array}{l}109.80 \\
(12.19)\end{array}$ & $\begin{array}{l}102.40 \\
(20.09)\end{array}$ & $9.927^{*}$ & 0.551 & 0.074 \\
\hline $\begin{array}{l}\text { SCWT Color } \\
\text { (C) }\end{array}$ & $\begin{array}{c}59.25 \\
(12.83)\end{array}$ & $\begin{array}{c}55.50 \\
(16.67)\end{array}$ & $\begin{array}{l}71.27 \\
(11.34)\end{array}$ & $\begin{array}{l}71.20 \\
(6.66)\end{array}$ & 1.139 & $6.222^{*}$ & 1.123 \\
\hline $\begin{array}{l}\text { SCWT Word- } \\
\text { Color (PC) }\end{array}$ & $\begin{array}{c}34.31 \\
(12.46)\end{array}$ & $\begin{array}{c}33.13 \\
(15.11)\end{array}$ & $\begin{array}{l}43.13 \\
(7.36)\end{array}$ & $\begin{array}{l}42.87 \\
(8.17)\end{array}$ & 0.485 & 1.662 & 0.266 \\
\hline
\end{tabular}




\begin{tabular}{|l|c|c|c|c|c|c|c|}
\hline \multicolumn{2}{|l|}{ Visuomotor tracking and cognitive flexibility } \\
\hline TMTA & $\begin{array}{c}48.81 \\
(28.25)\end{array}$ & $\begin{array}{c}55.50 \\
(39.43)\end{array}$ & $\begin{array}{c}37.93 \\
(12.30)\end{array}$ & $\begin{array}{c}41.2 \\
(16.97)\end{array}$ & 1.387 & 2.130 & 0.164 \\
\hline TMTB & $\begin{array}{c}146.13 \\
(104.29)\end{array}$ & $\begin{array}{c}158.88 \\
(124.63)\end{array}$ & $\begin{array}{c}87.87 \\
(29.25)\end{array}$ & $\begin{array}{c}80.47 \\
(25.40)\end{array}$ & 0.133 & $5.335^{\star}$ & 1.887 \\
\hline
\end{tabular}

The $F$ values refer to those obtained through a repeated-measures general linear model. $F(T)$ : time effect; $F(G)$ : group effect; $F(G \times T)$ : Interaction between time and group.

WM: working memory; RAVLT: Rey's auditory verbal learning test; SCWT: Stroop color-word test; TMTA: Trail-making test, part A; TMTB: Trail-making test, part B. 
Table 4. Volumetric data of subcortical gray matter structures, brainstem and ventricles

\begin{tabular}{|c|c|c|c|c|c|c|c|}
\hline & \multicolumn{2}{|c|}{$P D(n=16)$} & \multicolumn{2}{|c|}{ Controls $(n=15)$} & \multirow{2}{*}{$F(T)$} & \multirow{2}{*}{$F(G)$} & \multirow{2}{*}{$F(G \times T)$} \\
\hline & Baseline & Follow-up & Baseline & Follow-up & & & \\
\hline Thalamus & $\begin{array}{l}14.53 \\
(1.80)\end{array}$ & $\begin{array}{l}14.12 \\
(1.90)\end{array}$ & $\begin{array}{l}14.40 \\
(1.51)\end{array}$ & $\begin{array}{l}14.02 \\
(1.41)\end{array}$ & 0.014 & 0.004 & 0.022 \\
\hline Caudate & $\begin{array}{c}7.05 \\
(0.95) \\
\end{array}$ & $\begin{array}{c}6.77 \\
(0.91)\end{array}$ & $\begin{array}{c}7.46 \\
(1.27) \\
\end{array}$ & $\begin{array}{c}7.22 \\
(1.18)\end{array}$ & 0.883 & 2.583 & 0.184 \\
\hline Putamen & $\begin{array}{l}10.70 \\
(1.56)\end{array}$ & $\begin{array}{l}10.20 \\
(1.63)\end{array}$ & $\begin{array}{l}11.06 \\
(1.16)\end{array}$ & $\begin{array}{l}10.68 \\
(1.12)\end{array}$ & 1.640 & 1.277 & 0.855 \\
\hline Pallidum & $\begin{array}{c}3.35 \\
(0.46)\end{array}$ & $\begin{array}{c}3.37 \\
(0.42)\end{array}$ & $\begin{array}{c}3.34 \\
(0.40)\end{array}$ & $\begin{array}{c}3.36 \\
(0.41)\end{array}$ & 0.723 & 0.017 & 0.292 \\
\hline Hippocampus & $\begin{array}{c}8.76 \\
(1.02) \\
\end{array}$ & $\begin{array}{c}8.51 \\
(1.09) \\
\end{array}$ & $\begin{array}{c}8.58 \\
(1.13) \\
\end{array}$ & $\begin{array}{c}8.44 \\
(1.04) \\
\end{array}$ & 0.046 & 0.048 & 1.421 \\
\hline Amygdala & $\begin{array}{c}3.23 \\
(0.49) \\
\end{array}$ & $\begin{array}{c}3.07 \\
(0.50) \\
\end{array}$ & $\begin{array}{c}3.19 \\
(0.35)\end{array}$ & $\begin{array}{c}3.18 \\
(0.36)\end{array}$ & 0.098 & 0.132 & $5.661^{*}$ \\
\hline Accumbens & $\begin{array}{l}1.05 \\
(0.25)\end{array}$ & $\begin{array}{c}1.03 \\
(0.23)\end{array}$ & $\begin{array}{c}1.03 \\
(0.16)\end{array}$ & $\begin{array}{c}1.03 \\
(0.15)\end{array}$ & 2.559 & 0.004 & 0.458 \\
\hline Lateral ventricles & $\begin{array}{l}22.28 \\
(12.8)\end{array}$ & $\begin{array}{c}24.45 \\
(14.13)\end{array}$ & $\begin{array}{c}20.73 \\
(14.51)\end{array}$ & $\begin{array}{l}22.72 \\
(15.6)\end{array}$ & 1.542 & 0.037 & 0.020 \\
\hline $\begin{array}{c}\text { Inferior lateral } \\
\text { ventricles }\end{array}$ & $\begin{array}{c}0.75 \\
(0.34) \\
\end{array}$ & $\begin{array}{c}0.81 \\
(0.41)\end{array}$ & $\begin{array}{c}0.83 \\
(0.54)\end{array}$ & $\begin{array}{c}0.85 \\
(0.57)\end{array}$ & 0.243 & 0.218 & 1.492 \\
\hline 3rd ventricle & $\begin{array}{c}1.32 \\
(0.32)\end{array}$ & $\begin{array}{c}1.40 \\
(0.39)\end{array}$ & $\begin{array}{c}1.31 \\
(0.52)\end{array}$ & $\begin{array}{c}1.38 \\
(0.54)\end{array}$ & 0.698 & 0.002 & 0.001 \\
\hline 4 th ventricle & $\begin{array}{c}1.84 \\
(0.62) \\
\end{array}$ & $\begin{array}{c}1.81 \\
(0.59)\end{array}$ & $\begin{array}{c}2.00 \\
(0.48)\end{array}$ & $\begin{array}{c}2.04 \\
(0.51)\end{array}$ & 0.109 & 1.829 & 2.964 \\
\hline Brainstem & $\begin{array}{l}21.97 \\
(1.86)\end{array}$ & $\begin{array}{l}21.83 \\
(1.80\end{array}$ & $\begin{array}{l}22.59 \\
(2.56)\end{array}$ & $\begin{array}{l}22.47 \\
(2.58)\end{array}$ & 3.858 & 1.055 & 0.003 \\
\hline Cortex volume & $\begin{array}{l}455.65 \\
(53.3)\end{array}$ & $\begin{array}{l}444.14 \\
(47.46)\end{array}$ & $\begin{array}{l}450.88 \\
(42.09)\end{array}$ & $\begin{array}{l}449.30 \\
(38.41)\end{array}$ & $4.518^{\star}$ & 0.104 & $7.049^{\star}$ \\
\hline $\begin{array}{l}\text { Subcortical gray } \\
\text { matter volume }\end{array}$ & $\begin{array}{c}186.21 \\
(16.6)\end{array}$ & $\begin{array}{l}183.07 \\
(18.04) \\
\end{array}$ & $\begin{array}{l}182.23 \\
(16.66) \\
\end{array}$ & $\begin{array}{l}179.27 \\
(16.40) \\
\end{array}$ & 0.477 & 0.307 & 0.007 \\
\hline $\begin{array}{c}\text { Total gray matter } \\
\text { volume }\end{array}$ & $\begin{array}{c}641.86 \\
(64.1)\end{array}$ & $\begin{array}{l}627.21 \\
(60.30) \\
\end{array}$ & $\begin{array}{l}633.11 \\
(55.56) \\
\end{array}$ & $\begin{array}{l}628.57 \\
(51.72) \\
\end{array}$ & 4.100 & 0.002 & $5.400^{*}$ \\
\hline
\end{tabular}

The $\mathrm{F}$ values refer to those obtained through a repeated-measures general linear model (2x2 MANOVA): group factor (PD and Controls), time factor (baseline and followup). $F(T)$ : time effect; $F(G)$ : group effect; $F(G \times T)$ : Interaction between time and group. All the analyses were controlled by intracranial volume. ${ }^{*}$ significant at $p<0.05$. 
Table 5. Cortical areas showing significantly higher rates of thinning (mm/year) in PD patients compared to controls over time.

\begin{tabular}{|c|c|c|c|c|}
\hline Cortical Area & $\begin{array}{l}\text { Cluster } \\
\text { size } \\
(\mathrm{mm} 2)\end{array}$ & $\begin{array}{c}\text { Talairach } \\
\text { Coordinates of the } \\
\text { maxima } \\
x \quad y \quad z \\
\end{array}$ & $Z$ values & $\begin{array}{l}\text { Clusterwise } \\
\text { probability }(p)\end{array}$ \\
\hline \multicolumn{5}{|l|}{ Right hemisphere } \\
\hline $\begin{array}{l}\text { Superior frontal gyrus, } \\
\text { caudal middle frontal } \\
\text { region, precentral sulcus } \\
\text { and paracentral region }\end{array}$ & 2603.88 & $12.2-4.562 .7$ & 3.868 & $<0.001$ \\
\hline $\begin{array}{l}\text { Frontal pars opercularis, } \\
\text { precentral gyrus }\end{array}$ & 1737.77 & $\begin{array}{lll}47.4 & 12.3 & 15.3\end{array}$ & 3.074 & 0.001 \\
\hline $\begin{array}{l}\text { Superior temporal gyrus, } \\
\text { superior temporal } \\
\text { sulcus, supramarginal } \\
\text { region }\end{array}$ & 1197.26 & $43.9-4.2-16.2$ & 5.115 & $<0.001$ \\
\hline \multicolumn{5}{|l|}{ Left hemisphere } \\
\hline $\begin{array}{l}\text { Caudal middle frontal, } \\
\text { precentral, rostral middle } \\
\text { frontal gyrus }\end{array}$ & 1373.34 & $\begin{array}{lll}-34.6 & 23.0 & 43.5\end{array}$ & 3.100 & $<0.005$ \\
\hline $\begin{array}{l}\text { Middle temporal gyrus, } \\
\text { superior temporal gyrus, } \\
\text { superior temporal } \\
\text { sulcus, inferior parietal } \\
\text { lobe }\end{array}$ & 1062.84 & $-59.4-49.64 .1$ & 2.511 & 0.026 \\
\hline
\end{tabular}

The results were corrected using family wise error correction with Z Monte Carlo simulation (10000 iterations) and thresholded at a corrected $p$ value of $0.05(Z=1.3)$. 


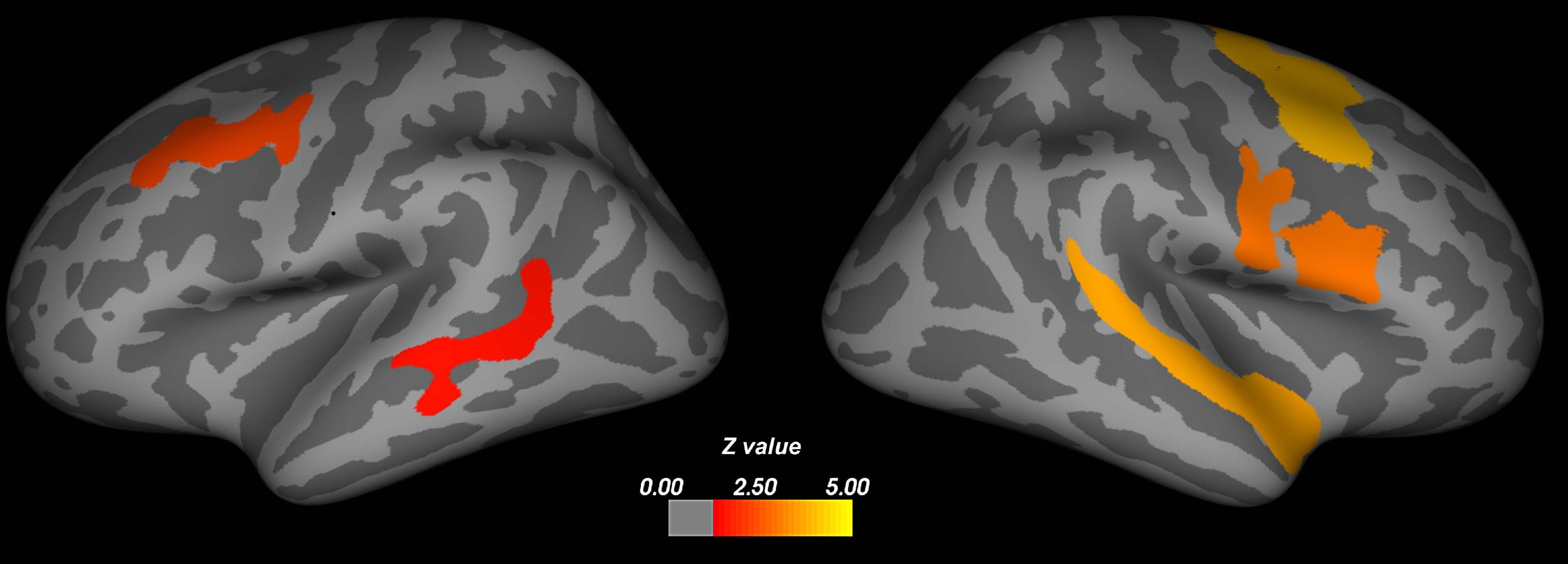




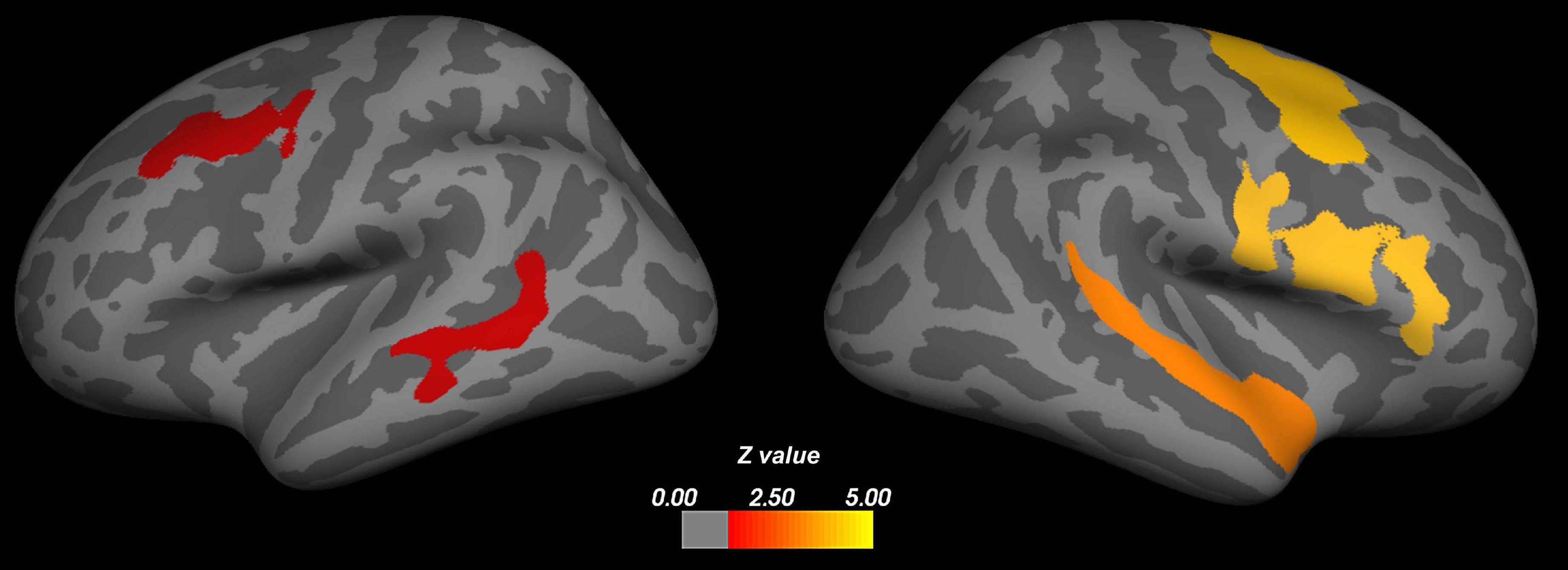

